\title{
High power disk lasers: advantages and prospects
}

\author{
V. V. Apollonov \\ Prokhorov General physics Institute RAS, Vavilova 38, Moscow
}

Email address:

vapollo@kapella.gpi.ru (V. V. Apollonov)

\section{To cite this article:}

V. V. Apollonov. High Power Disk Lasers: Advantages and Prospects. American Journal of Modern Physics. Vol. 1, No. 1, 2012, pp. 1-6. doi: 10.11648/j.ajmp.20120101.11

\begin{abstract}
The mono-module disk laser concept is an effective design for diode-pumped solid-state lasers, which allows the realization of lasers with super-high output power, having very good efficiency and also excellent beam quality. Since the first demonstration of the principle in 1966 [1] the output power of mono-module disk has been increased to the level of few $\mathrm{kW}$ in continuous wave (CW) mode of operation. "Zig-Zag" disk laser geometry does not look like as a perspective one for further output parameters growing. The scaling laws for mono-module disk laser design show that the limits for CW mode of operation is far beyond $100 \mathrm{~kW}$ for output power and the energy can be higher than $100 \mathrm{~J}$ in pulsed mode of operation. Due to the efficient porous cooling technology and possibility of amplified spontaneous emission (ASE) suppression the operation of the mono-module disk laser geometry is possible in CW and pulse-periodical (P-P) modes at extremely high output power.
\end{abstract}

Keywords: Disk Laser, Efficient Porous Cooling, Amplified Spontaneous Emission, “Zig-Zag” Geometry, Electrical Discharge CO2-Lasers, Ablation, Pulse-Periodical Mode Of Operation

\section{Introduction}

It has long been customary that as new technologies emerge into the light, potential users and experts start wondering whether these new techs will replace the old, well-established approaches to the solution of known problems. When we look back at the time when the first laser was created, it becomes clear that each new and more advanced technology usually replaces the pre-existing and well-proven technologies. However, a more differentiated and balanced assessment of the many innovations in the field of laser shows that there is no such thing as a perfect design, or an ideal laser. There is always room for improvement and further development. It is possible that in the near future a laser with the disk geometry of the active medium will become the dominant technology. However, despite this, a number of existing laser technologies (with some exceptions, of course) will continue to improve for quite a long time. Based on these considerations, we must continue to develop most types of lasers, each time clearly specifying their undeniable technological niche and knowing well their advantages and disadvantages.

High efficiency and excellent beam quality of disk lasers mean that they can be widely used in modern science and industry for a very large range of applications, including treatment of the surface of dielectric materials in microelectronics, cutting, drilling, welding, polishing and clean- ing of the surface, other technological operations with superhard and fragile metals and composite materials, etc. Pulse-periodic Q-switched disk lasers and high-average-power, mode-locked laser systems ensure optimal conditions for ablation (sublimation) of a material. A number of advantages of high repetition rate P-P lasers emitting short pulses, as applied to a wide range of industrial technologies, is the basis of many modern concepts of disk lasers. High-intensity light with an insignificant thermal lens effect in the central high-loaded zone of the active medium have led to the lifting of restrictions on the brightness of the pump diode. This has reduced the cost of laser sources, and thus has significantly improved the efficiency of electro-optical conversion, especially in the regime of high average power. The power of the laser source is varied by scaling the cross sectional area of the generated radiation. It should be noted that its ratio to the thickness of the active material in the disk geometry is much larger than the ratio of the cross section of typical core elements of the active medium to their length in any conventional solid-state laser systems. This eliminates the problems associated with nonlinear distortions of the geometry of the active medium, and enables realizing superhigh peak values and average powers of laser sources with the disk geometry and the same parameters of the radiation in the far-field region. 


\section{Disk and Fiber Lasers}

It is clear that each laser source has its advantages and disadvantages. Beam quality of modern solid-state lasers (disk and fiber lasers) is pretty good, and their efficiency is very high. A fiber laser has significant advantages at low, average and peak output powers, while a disk laser, on the contrary, is preferable in those applications which require high and super-high output powers.

Among high repetition rate P-P sources emitting coherent high average and peak output radiation, disk lasers will undoubtedly dominate today and in the future. They provide the best solution for many industrial applications in the multi-kilowatt power range. A disk is a simple and easily excited laser element, which allows cost-effective generation of radiation with improved parameters. It has a lot of potential for the future. The advantage of the disk laser over the fiber laser is already obvious in the kilowatt power range. Having a large radiating surface, the power density of the disk laser is not critical even at high peak powers. On the contrary, in fiber lasers the increase in the peak power negatively affects the reliability of the resonator. The main disadvantage of the fiber laser is also its high sensitivity to the reflected beam, which often appears in the laser due to the interaction of the generated light with matter. If the radiation reflected from the target affects the fiber laser resonator, then it should be immediately shut down to preserve the cavity.

At the same time, the disk laser resonator is insensitive to the penetration of reflected beams. Even in the case of highly reflective materials, technological operations can be performed without the risk of an emergency switching off of the laser. Another advantage of kilowatt disk lasers is their modular design. This design allows one to replace laser modules during after-sales service, thereby ensuring that in the event of failure of the laser, the downtime as well as the cost of repairs will be minimal.

On the contrary, in fiber lasers, the single-unit design of the resonator makes it impossible to replace easily the failed unit without extensive alteration of the system as a whole. At the same time, the use of fiber laser technology offers certain advantages, especially in the power range (up to several hundreds of watts). This laser is suitable for micromachining of dielectric surfaces and composite materials. However, only the disk laser has a high potential in the case of high peak powers, i.e., when it is necessary to provide a train of short pulses with the highest peak power. In a typical disk laser, as opposed to a fiber laser, the power density inside the cavity is far below the critical damage threshold of the laser active medium and surfaces of auxiliary optical elements, even at a high peak power. For this reason, a disk laser allows, among other things, a better use of the laser medium in general, and significantly improvement in laser efficiency.

Thus, in the future solid-state diode-pumped disk lasers will play the dominant role. Laser diode structures are central elements in many new types of lasers. Today, the decisive factor is the acquisition of the necessary experience and skills to design most efficiently new laser systems. Semiconductor-based technologies and applications of laser equipment on new physical principles are key technologies of modern times. For many years, the world has accumulated the experience needed today in this field of knowledge. The challenge is to ensure technological excellence in the application of laser systems, not only today but in the future.

\section{Design and Physical Foundations of Disk Laser Operation}

The principle of the disk laser operation is based on the use of an active element in the form of a disk with a cooled surface. High cooling efficiency of the laser medium is provided by a large area of the disk surface, which is important from the point of view of the heat transfer process. Therefore, the average power in the beam can reach quite high values. It is important to note that due to effective heat transfer within the disk area there is no thermal lens effect, characteristic of 'rod' and 'slab' (optical range) geometries of the active element. In this case, cooling is performed through a side surface of the active element; a two-dimensional heat flow, forming a parabolic profile of thermal distortion, propagates through this side surface. The temperature dependence of the refractive index in this case leads to the emergence of a strong thermal lens effect. Such a lens deteriorates the directivity of the output and significantly limits the effectiveness of its action. The thin disk, in contrast, is cooled through a thin contact region on one side and generates a one-dimensional axial heat flow on the other side. As a result, the temperature gradient is distributed parallel to the laser beam, which does not result in the thermal lens effect. In practice, a thin laser element in high-power disk lasers is either connected to a porous heat sink or is cooled by forced convection [2,3].

In both cases, the undesirable thermal lenses are eliminated by the optimum layout of structural elements of the disk laser. Integral heat transfer from the active material to the porous heat sink depends directly on the area of the pump zone and on the geometry of the heat transfer contact. At a constant temperature of the active laser element, the average output power achieved is directly proportional to the area of the pump zone and, consequently, to the cross-sectional area of the laser beam on the disk. This is a unique feature of disk lasers, which allows one to change the power density at a constant energy of the beam on the disk. This property is particularly important for multi-kilowatt sources, because it makes it possible to adjust the value of the peak output power without changing many other parameters that affect the performance of the overall system.

At first glance, the basic challenge is the disk thickness needed for efficient heat dissipation, because the conventional scheme involves low absorbance of the active disk material. However, the pump scheme allows one to find an elegant solution to this problem. The process of excitation in the active material of the disk can be represented in the form 
of multi-channel optical configuration [1,4] consisting of a parabolic mirror and a pump deflection system. The parabolic mirror focuses a collimated beam of the laser cavity containing an active element. After partial absorption of radiation passing through the disk body, some energy of the beam is reflected from a highly reflective mirror located on the backside of the disk. As a result of multiple reflections through prisms and the parabolic mirror in general we deal with 20 iterations of radiation passes through the body of the disk, which ensures high efficiency of absorption of light energy of the pump beam. The optical scheme of such resonators tolerates limitations on the brightness of the pump source. Generally, pumping of a high average power disk laser does not require a beam of high optical quality. That is why both laser diodes and rather complex laser diode structures with a uniform intensity distribution can be used in this case. In terms of the practical use laser diodes with a uniform intensity distribution are more preferable due to much lower energy consumption per one watt of the pump power.

For efficient processing of materials: cutting, drilling, polishing and removal of a thin film from the surface of various materials laser pulses with a high peak power and high repetition rate are needed. Typical pulse duration of a Q-switched Yb:YAG disk laser lies within one microsecond. Q-switching is provided by means of an acousto-optic modulator. Depending on the resonator geometry it is possible to generate pulses with duration from a few hundred picoseconds to a microsecond. To date, the best results achieved with the help of P-P disk lasers having high average power are as follows: pulse repetition rate - up to $10 \mathrm{kHz}$; maximum pulse energy - $100 \mathrm{~mJ}$; peak power - $200 \mathrm{~kW}$; average power - up to $1000 \mathrm{~W}$. However, there are fundamental physical limitations on further scaling of the disk lasers, which we will discuss below.

\section{Laws of Scaling of Disk Lasers}

The power of disk lasers is limited not only by the power of the pump and overheating of the medium but also by the losses due to the ASE and the background radiation losses in the resonator. To avoid overheating, the size of the active medium should vary in accordance with the law of power scaling. Then, to avoid large losses caused by their exponential growth during the ASE, amplification of radiation corresponding to transverse round trips should not be large. This requires a reduction in gain G. Gain is determined by the reflectivity of the output mirror and disk thickness. Amplification of radiation per round trip, however, should not be substantially greater in magnitude than the radiation loss per round trip along the same optical path. The difference between the gain and losses per round trip of radiation determines the optical energy that is coupled out from the laser cavity. Reduction of the gain at this loss level requires an increase in the disk thickness. In this case, at a certain critical size, the disk becomes optically too thick and cannot be pumped above the threshold without overheating. Some features of scaling can be shown on a simple model. Suppose $\mathrm{M}$ is the saturation intensity of the medium. The corresponding optimal thickness of the disk can be estimated as $\mathrm{h} \sim \mathrm{T} / \mathrm{Mb}$. The corresponding optimal lateral dimensions of the disk can be represented in the form: $\mathrm{D} \sim \mathrm{T} / \mathrm{Mb} 2$, where $\mathrm{T}$ is a parameter of the heat load. Roughly speaking, the loss per round trip of radiation must be scaled inversely proportional to the cube root of the power required: $\mathrm{P} \sim \mathrm{n} \mathrm{T2} / \mathrm{M}$ b3.

An additional problem is the efficient delivery of pump energy. In cases with a small-signal gain, single-pass absorption of the pump is also low. It follows that the effective utilization of the pump energy is very necessary for the effective operation of the laser disk. To scale the output power, the medium must be optically thin, which requires a large number of passes of the pump energy through the medium. Besides, the pump energy coupled in through the lateral side of the disk can also be a possible solution for efficient pumping.

To reduce the effects of the ASE, it was proposed to use an optical cover consisting of an undoped material on the surface of the laser disk. This cover allows the spontaneously emitted photons to escape from the active layer and prevents their resonance in the bulk of active material. Rays cannot be reflected from the surface, as in the case of an open disk. This allows the maximum power achievable by the disk laser to be increased by an order of magnitude. Reflection of the ASE from the disk edge should also be suppressed. This can be done through the absorbing layer at the generatrix of the disk cylinder. In the regime when output power is close to maximal, much of the energy is used in the ASE; therefore, the absorbing layers must also have radiators accumulating heat. In the case of the maximum pump density of the disk laser its efficiency is quite low: Most of the pump power is used in the ASE and is absorbed at the edges of the device. In this case, the distribution of the pump energy between several disks can significantly improve the performance of the laser system. Indeed, lasers, consisting of several modules with disk elements in a single cavity, have been repeatedly reported. One of these lasers, fabricated by the TRUMPF Group [4].

- a world leader in this class of laser systems - is presented at Fig. 1.

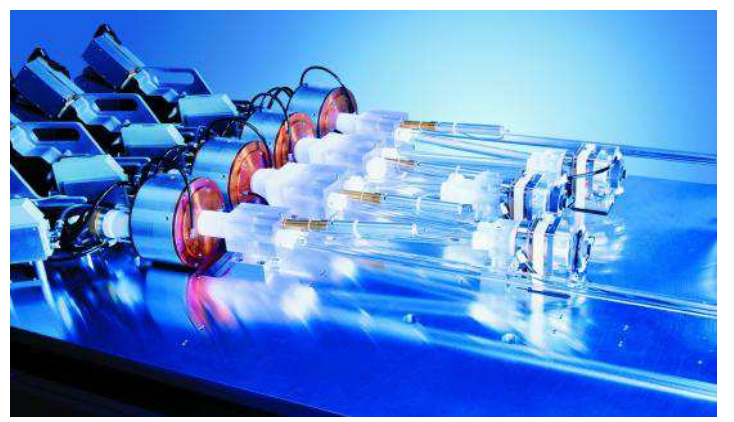

Fig. 1. "Zig-Zag" disk Laser consisting of a series of disk modules in a single cavity (TRUMPF [2]).

In the history of electrical discharge CO2-lasers output 
power scaling a very similar geometry had been investigated carefully. This is a "Zig-Zag"-CO2 laser which was created in FIAN by A.I.Barchukov in the beginning of 70`s. The future of "Zig-Zag" multi- module geometry disk laser probably will be the same. At the Fig. 2 "Zig-Zag"CO2-laser created by A.I. Barchukov FIAN 1970 [3] is presented. With the help of this laser we have forecasted a lot of new effects which later on made significant difficulties to the scientists involved into high power mono-module laser creation. There are: deformation of the high power laser mirrors surface and distortion of phase front of the beam during propagation and as the result-high power static and adaptive optics, big scale water cooled optics for telescopes based on $\mathrm{SiC}$, high power $\mathrm{CW}$ plasmotrone in air and as the result- crusibleless growing of crystals, high quality laser beam and as the result- high quality laser based hard materials welding and cutting and so on.

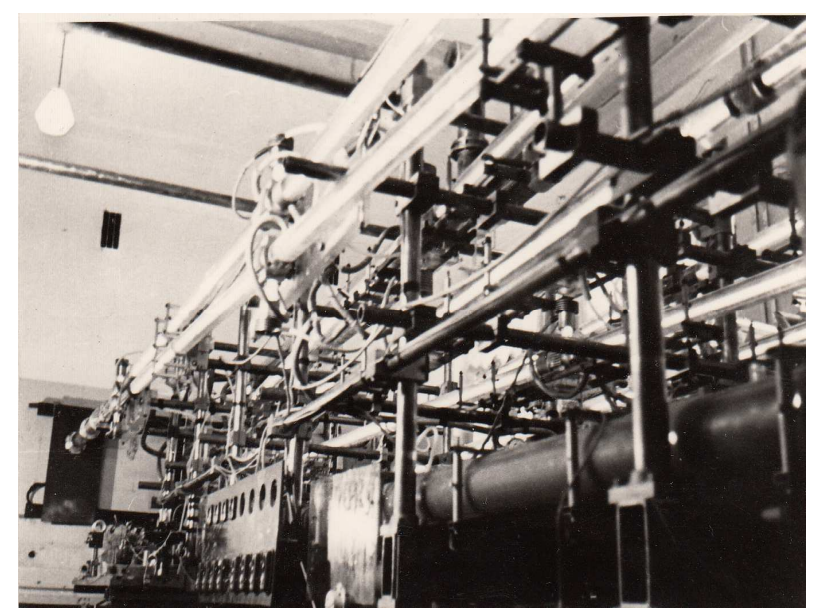

Fig. 2. "Zig-Zag"- CO2-laser created by A.I. Barchukov FIAN 1970 [2].

In the quasi-continuous regime, the power can be estimated by scaling the saturation intensity with a duty cycle of the pump and by multiplying the duration of the pump by the pulse repetition rate. At moderate repetition rates (e.g., higher than $1 \mathrm{~Hz}$ ), the maximum energy of the output pulses is approximately inversely proportional to the cube of background losses $b$. The undoped cover can increase the average output power by an additional order of magnitude, provided that this cover does not increase the background loss. At low pulse repetition rates (in the single-pulse regime) and sufficient pump power, there is no general limitation on the energy, but the required size of the device increases rapidly with increasing pulse energy, thereby establishing a practical limit of energy. One active element, according to the estimates, can generate an optical pulse with energy of a few thousand joules, depending on the level of internal signal loss in the disk.

Disk lasers, as well as fiber lasers, have a large ratio of the cooling surface area to the gain of the laser. However, these two different concepts of the laser design also differ by the values of the achievable peak power. Beam quality of fiber lasers is determined by properties of waveguides, the refractive index difference between the core and the cladding, and the size of the internal diameter of the fiber that transmits light. On the other hand, beam quality of disk lasers depends on the design of the resonator. With increasing size of the optical pump region (its diameter is usually a few millimeters) at a constant optical power density on the disk surface, scaling of the output power becomes possible. Adjustment of the resonator also has similar features. Self-phase modulation defines the typical limit of nonlinear amplification of ultrashort pulses. It leads to an extension of the spectral line proportional to the ratio of the effective optical path in the material with nonlinear characteristics to the effective cross-sectional area of the beam multiplied by the square of the pulse duration. An electro-optical switch in a regenerative amplifier of the disk laser makes a substantial contribution to the nonlinear characteristics of the gain which is greater than the contribution from the disk. It must be emphasized that the values of the peak power, achieved today in single disk modules, are much smaller than the values obtained in 'rod' and 'slab' solid-state laser systems. However, the level of the average power generated by a single disk module also leaves much to be desired.

\section{Regenerative Amplification of Pulses}

At present, in the scientific and technical research and manufacturing processes relying on the use of high average power lasers, only sources operating in two regimes find applications, i.e., CW and P-P laser sources with a pulse repetition rate up to hundreds of $\mathrm{Hz}$ and pulse duration from tens of microseconds to tens of milliseconds. In most processes, purely thermal mechanism of action is mainly implemented, since use is made of the possibility of a laser source to deliver quite a lot of energy to a small area of the surface of the work piece. High-frequency, high-average-power laser systems operating in the Q-switched regime, which provides the pulse duration in a periodic train from a few to hundreds of nanoseconds, allows for a fundamentally different mechanism of interaction of radiation with matter, i.e., ablation, ensuring local release of energy not only in space but also in time [5]. This results in an explosive local evaporation of the substance without an intermediate liquid phase. This mechanism can significantly extend the range of technological applications of laser sources. Today, it has found a real use in practice, only at an insignificant level of output power in the range of $1000 \mathrm{~W}$. However, there arises a reasonable question: Why are high-power P-P lasers not available at the international laser market? The answer is simple: The complexity of their manufacturing consists in the inability of application of the classical modulation methods to laser systems with high average and peak powers. At the same time, it is quite clear that the creation of high-power $(>1 \mathrm{~kW})$ P-P lasers with frequencies up to $100 \mathrm{kHz}$ or higher (with a peak power exceeding the average power by two to three orders of magnitude) would significantly expand the field of application of laser sources, increase their efficiency, and implement qualitatively new effects[6]. 
One of the successful trends in implementation of new methods of high-frequency modulation of the output from high-power laser sources is due to the regenerative amplification of a small signal injected into the cavity of a high-power gas or solid-state laser with the classical rod geometry. Use of intracavity modulators in high-power laser systems is hindered, because high power density inside the cavity leads to the appearance of plasma on the surface elements of the modulator, to the screening of radiation and to the destruction of the optical elements of the modulator. Quite promising is the method of realization of the P-P regime in high-power lasers, which is based on the use a self-filtering cavity. This cavity is a confocal resonator consisting of two spherical mirrors of different curvatures; in the common focal plane there is an annular output mirror with a hole. Because of the high degree of discrimination of the higher modes with respect to the resonator losses, the lower mode is reliably generated. The spherical resonator mirror is placed outside the active medium and contains a modulation unit comprising spherical mirrors. The laser mode should be formed quickly enough in the leading edge of a giant pulse. The problem of laser micromachining usually involves cutting, drilling, surface cleaning and polishing, as well as removal of thin layers due to the ablation of the material. Micromachining relies on lasers emitting pulses of different duration. The geometry of the beam and the pulse repetition rate are crucial: usually the pulse duration does not exceed a few tens of nanoseconds, and in some cases reaches several hundreds of picoseconds. Disk laser technology provides excellent flexibility in the scaling of the output power by combining it with a high pulse energy and excellent beam quality.

\section{Prospects for Scaling the Power of Disk Lasers}

As mentioned above, the design of the disk laser generating a continuous and relatively high power is ideal for cutting and welding metals, where high optical quality of the beam is required. It is important for such industries as automotive, transportation, aerospace and heavy engineering. However, the design of the disk laser is more suitable for a range of new technologies that are currently in demand. Today, a multi-module laser with a power up to $16 \mathrm{~kW}$ and beam quality of $\geq 2 \mathrm{~mm}$ mrad, fabricated by the TRUMPF Group, is the undisputed leader in this class of laser systems. CW solid-state diode-pumped disk lasers demonstrate highly efficiency. Beam quality of the laser disk is outstanding, which makes it possible to work with a target from a large distance, while providing an extremely high concentration of radiation in the interaction region with the help of the focusing optics. In this design of the laser module the disk size is no more than 4-5 mm, because at larger sizes the energy loss due to the amplified spontaneous emission increases at an absolutely unacceptable rate. The above-presented laser system consists of a set of disk mod- ules in a single cavity. This geometry of the laser system allows implementation of the P-P regime with a fairly high peak power, but the average power lies within a kilowatt. Parallel operation of disk lasers can increase the peak power of the entire system, but the phase-locking of the disk channels in the P-P regime requires additional scientific research. Further expansion of the power to the megawatt level of both the average and peak power in both geometries is very problematic.

At the same time, a different approach is known to the implementation of scalable solid-state laser systems, which consists of a set of active elements in the form of 'slabs', followed by phase-locking of the generated radiation. The team of the Northrop Grumman Corporation has created a laser with a power of $>100 \mathrm{~kW}$ and high laser beam quality equal to the diffraction limit of 1.5 (averaged value) with the exposure time of 300 seconds [7]. The laser efficiency reaches $30 \%$. The authors of the project point to the easy replacement of individual laser channels in case of failure. They also mentions some of the advantages of the parallel structure of the amplifying channels in terms of ease of further increasing output power, if necessary, to $100 \mathrm{~kW}$. Beside, the laser assembled according to this scheme provides the divergence at the level of two diffraction limits from the common (composite) aperture. From general physical considerations, we can assume that for a given power level and a reasonable value of radiation resistance of optical elements, the divergence of the laser radiation at the level of $2 \times 10-5 \mathrm{rad}$ can be achieved for the $\mathrm{CW}$ generation regime and $0.6 \times 10-5$ rad for the P-P regime. A further increase in the number of channels in order to obtain an output power of $1 \mathrm{MW}$ will require the coherent summation of the power from at least 80 channels, which seems an elusive task.

The question arises as to how the average power of a few megawatts can be achieved on the basis of solid-state lasers. And it is this power that is needed to address many problems associated with the removal of debris from near-Earth space, with launching of missiles with the help of lasers, with the creation of long-range conducting channels and others. Fiber lasers are not applicable for these purposes because of the smallness of the area of the exit pupil of the fibers and hence the impossibility of operation of such lasers in the high-frequency P-P regime with high peak power at an average power of a few megawatts [8-9]. The laser system based on 'slabs' also seems hard to implement as adjustment of the system and its maintenance in a safe operation mode are comparable to the complexity of working with a multi-element system for the solution to the problem of controlled thermonuclear fusion (CTF) at Livermore (USA). Proceeding from the above, the answer is quite clear: Such a laser can and should be based on the mono-module disk laser geometry!

This is due to the indisputable advantages of the disk geometry in terms of the minimal thermal lens in the active media and the high radiation resistance of the disk in the P-P regime because of the large area of the optical surface to 
couple out the radiation. So, the necessity to find a solution to the problem of the ASE suppression along the diameter of the disk was the major problem (matter of patent). In our case, the size of the disk at a megawatt level of the average power output should be at least $50 \mathrm{~cm}$, i.e., at least hundred times bigger the size of the disk that is used today in existing systems. Radiation from such a laser, obtained during generation in the active medium of a single disk, does not require additional phase-locking. At the same time, such a laser in a mono- module geometry will be very well combined with a large-diameter telescope for ensuring high peak power density of the laser pulse on space debris. It is known that the disk geometry of a laser was proposed 46 years ago; however, to this day, the solution to the problem of the ASE suppression with increasing transverse dimensions of the active medium in the mono-module disk geometry is found! Thus, the prospects of new versions of the mono-module disk laser creation for new class of cutting edge problems are open!

\section{Conclusions}

Only the future will show the final physical-technical solution for the mono-module disk laser which will be used effectively for the processing of materials and for the solution of other important challenges faced by science and technological advances of the future. However, today we can say that creation of megawatt class high repetition rate P-P disk lasers with a large cross section of the active medium will open up great prospects for their use in solving the problems of launching of small satellites with lasers, for- mation of super-long conducting channels in space and atmosphere, cleaning of the near-Earth space from the space debris, etc. [10-12].

\section{Reference}

[1] Basov N.G., Bogdankevich O.V., Grasiuk A.Z. IEEE J. of , QE-2 (9), (1966).

[2] V.V. Apollonov, A.I.Barchukov, A.M.Prokhorov, JEEE J. of QE-6, 10, (1974), Report of FIAN, (1971).

[3] V.V. Apollonov, A. M. Prokhorov, A. H. Guenther, Laser Physics V. 11, N.8, (2001).

[4] SPIE Events Europe, TRUMPF`s R@D, (2012).

[5] V.V. Apollonov, P.B.Corkum, R.S.Teylor, Appl.Phys.Lett., 35,147, (1979).

[6] V.V. Apollonov,V.I. Kislov, V.V. Kijko, A.G. Suzdal'tsev, QE,33 (9), (2003).

[7] SPIE Events Europe, Nothrop`s R@D, (2012).

[8] V.V. Apollonov, Symposium HPLS@A-2012, Istanbul 10 September (2012).

[9] V. V. Apollonov, V. I. Kislov, A. M. Prokhorov, QE 23(5), (1996).

[10] V.V. Apollonov, Natural science, 4, 713, (2012).

[11] V.V. Apollonov, Natural science, 4, 719, (2012).

[12] V.V.Apollonov, Natural science, 4, 725, (2012). 